\title{
A GENERAL FRAMEWORK FOR ISLAND SYSTEMS
}

\author{
STEPHAN FOLDES, ESZTER K. HORVÁTH, SÁNDOR RADELECZKI, \\ AND TAMÁS WALDHAUSER
}

\begin{abstract}
The notion of an island defined on a rectangular board is an elementary combinatorial concept that occurred first in 3 . Results of 3 were starting points for investigations exploring several variations and various aspects of this notion.

In this paper we introduce a general framework for islands that subsumes all earlier studied concepts of islands on finite boards, moreover we show that the prime implicants of a Boolean function, the formal concepts of a formal context, convex subgraphs of a simple graph, and some particular subsets of a projective plane also fit into this framework.

We axiomatize those cases where islands have the property of being pairwise comparable or disjoint, or they are distant, introducing the notion of a connective island domain and of a proximity domain, respectively. In the general case the maximal systems of islands are characterised by using the concept of an admissible system. We also characterise all possible island systems in the case of connective island domains and proximity domains.
\end{abstract}

\section{INTRODUCTION}

"ISLAND, in physical geography, a term generally definable as a piece of land surrounded by water." (Encyclopædia Britannica, Eleventh Edition, Volume XIV, Cambridge University Press 1910.) Mathematical models of this definition were introduced and studied by several authors. These investigations utilized tools from different areas of mathematics, e.g. combinatorics, coding theory, lattice theory, analysis, fuzzy mathematics. Our goal is to provide a general setting that unifies these approaches. This general framework encompasses prime implicants of Boolean functions and concepts of a formal context as special cases, and it has close connections to graph theory and to proximity spaces.

The notion of an island as a mathematical concept occurred first in Czédli [3], where a rectangular board was considered with a real number assigned to each cell of the board, representing the height of that cell. A set $S$ of cells forming a rectangle is called an island, if the minimum height of $S$ is greater than the height of any cell around the perimeter of $S$, since in this case $S$ can become a piece of land surrounded by water after a flood producing an appropriate water level. The motivation to investigate such islands comes from Foldes and Singhi [9], where islands on a $1 \times n$ board (so-called full segments) played a key role in characterizing maximal instantaneous codes.

The main result of $[3$ is that the maximum number of islands on an $m \times n$ board is $\lfloor(m n+m+n-1) / 2\rfloor$. However, the size of a system of islands (i.e., the collection of all islands appearing for given heights) that is maximal with respect to inclusion (not with respect to cardinality) can be as low as $m+n-1$ [18]. Another important observation of [3] is that any two islands are either comparable (i.e. one is contained in the other) or disjoint; moreover, disjoint islands cannot be too close to each other

2010 Mathematics Subject Classification. 06A06.

Key words and phrases. Island system, height function, CD-independent and CDW-independent sets, admissible system, distant system, island domain, proximity domain, point-to-set proximity relation, prime implicant, formal concept, convex subgraph, connected subgraph, projective plane. 
(i.e. they cannot have neighboring cells). It was also shown in [3] that these properties actually characterize systems of islands. We refer to such a result as a "dry" characterization, since it describes systems of islands in terms of intrinsic conditions, without referring to heights and water levels.

The above mentioned paper [3] of Gábor Czédli was a starting point for many investigations exploring several variations and various aspects of islands. Square islands on a rectangular board have been considered in [15, 20, and islands have been studied also on cylindrical and toroidal boards [1, on triangular boards [14, 19, on higher dimensional rectangular boards [24] as well as in a continuous setting [21, 25]. If we allow only a given finite subset of the reals as possible heights, then the problem of determining the maximum number of islands becomes considerably more difficult; see, e.g. [13, 17, 22. Islands also appear naturally as cuts of lattice-valued functions [16; furthermore, order-theoretic properties of systems of islands proved to be of interest on their own, and they have been investigated in lattices and partially ordered sets [4, 6, 12. The notion of an island is an elementary combinatorial concept, yet it leads immediately to open problems, therefore it is a suitable topic to introduce students to mathematical research 23$]$.

In this paper we introduce a general framework for islands that subsumes all of the earlier studied concepts of islands on finite boards. We will axiomatize those situations where islands have the "comparable or disjoint" property mentioned above, and we will also present dry characterizations of systems of islands.

\section{Definitions AND EXAMPles}

Our landscape is given by a nonempty base set $U$, and a function $h: U \rightarrow \mathbb{R}$ that assigns to each point $u \in U$ its height $h(u)$. If the minimum height $\min h(S):=$ $\min \{h(u): u \in S\}$ of a set $S \subseteq U$ is greater than the height of its surroundings, then $S$ can become an island if the water level is just below $\min h(S)$. To make this more precise, let us fix two families of sets $\mathcal{C}, \mathcal{K} \subseteq \mathcal{P}(U)$, where $\mathcal{P}(U)$ denotes the power set of $U$. We do not allow islands of arbitrary "shapes": only sets belonging to $\mathcal{C}$ are considered as candidates for being islands, and the members of $\mathcal{K}$ describe the "surroundings" of these sets.

Definition 2.1. An island domain is a pair $(\mathcal{C}, \mathcal{K})$, where $\mathcal{C} \subseteq \mathcal{K} \subseteq \mathcal{P}(U)$ for some nonempty finite set $U$ such that $U \in \mathcal{C}$. By a height function we mean a map $h: U \rightarrow$ $\mathbb{R}$.

Throughout the paper we will always implicitly assume that $(\mathcal{C}, \mathcal{K})$ is an island domain. We denote the cover relation of the poset $(\mathcal{K}, \subseteq)$ by $\prec$, and we write $K_{1} \preceq K_{2}$ if $K_{1} \prec K_{2}$ or $K_{1}=K_{2}$.

Definition 2.2. Let $(\mathcal{C}, \mathcal{K})$ be an island domain, let $h: U \rightarrow \mathbb{R}$ be a height function and let $S \in \mathcal{C}$ be a nonempty set.

(i) We say that $S$ is a pre-island with respect to the triple $(\mathcal{C}, \mathcal{K}, h)$, if every $K \in \mathcal{K}$ with $S \prec K$ satisfies

$$
\min h(K)<\min h(S) .
$$

(ii) We say that $S$ is an island with respect to the triple $(\mathcal{C}, \mathcal{K}, h)$, if every $K \in \mathcal{K}$ with $S \prec K$ satisfies

$$
h(u)<\min h(S) \text { for all } u \in K \backslash S .
$$

The system of (pre-)islands corresponding to $(\mathcal{C}, \mathcal{K}, h)$ is the set

$$
\{S \in \mathcal{C} \backslash\{\emptyset\}: S \text { is a (pre-)island w.r.t. }(\mathcal{C}, \mathcal{K}, h)\} .
$$


By a system of (pre-)islands corresponding to $(\mathcal{C}, \mathcal{K})$ we mean a set $\mathcal{S} \subseteq \mathcal{C}$ such that there is a height function $h: U \rightarrow \mathbb{R}$ so that the system of (pre-)islands corresponding to $(\mathcal{C}, \mathcal{K}, h)$ is $\mathcal{S}$.

Remark 2.3. Let us make some simple observations concerning the above definition.

(a) Every nonempty set $S$ in $\mathcal{C}$ is in fact an island for some height function $h$.

(b) If $S$ is an island with respect to $(\mathcal{C}, \mathcal{K}, h)$, then $S$ is also a pre-island with respect to $(\mathcal{C}, \mathcal{K}, h)$. The converse is not true in general; however, if for every nonempty $C \in \mathcal{C}$ and $K \in \mathcal{K}$ with $C \prec K$ we have $|K \backslash C|=1$, then the two notions coincide.

(c) The set $U$ is always a (pre-)island. If $S$ is a (pre-)island that is different from $U$, then we say that $S$ is a proper (pre-)island.

(d) If $S$ is a pre-island with respect to $(\mathcal{C}, \mathcal{K}, h)$, then the inequality $\min h(K)<$ $\min h(S)$ of (i) holds for all $K \in \mathcal{K}$ with $S \subset K$ (not just for covers of $S$ ).

(e) Let $\mathcal{C} \subseteq \mathcal{K}^{\prime} \subseteq \mathcal{K}$. It is easy to see that any $\mathcal{S} \in \mathcal{C}$ which is a pre-island with respect to the triple $(\mathcal{C}, \mathcal{K}, h)$ is also a pre-island with respect to $\left(\mathcal{C}, \mathcal{K}^{\prime}, h\right)$.

(f) The numerical values of the height function $h$ are not important; only the partial ordering that $h$ establishes on $U$ is relevant. In particular, one could assume without loss of generality that the range of $h$ is contained in the set $\{0,1, \ldots,|U|-1\}$.

Many of the previously studied island concepts can be interpreted in terms of graphs as follows.

Example 2.4. Let $G=(U, E)$ be a connected simple graph with vertex set $U$ and edge set $E$; let $\mathcal{K}$ consist of the connected subsets of $U$, and let $\mathcal{C} \subseteq \mathcal{K}$ such that $U \in \mathcal{C}$. In this case the second item of Remark 2.3 applies, hence pre-islands and islands are the same. Let us assume that $G$ is connected, and let $\mathcal{C}$ consist of the connected convex sets of vertices. (A set is called convex if it contains all shortest paths between any two of its vertices.) If $G$ is a path, then the islands are exactly the full segments considered in [9], and if $G$ is a square grid (the product of two paths), then we obtain the rectangular islands of [3]. Square islands on a rectangular board [15, 20], islands on cylindrical and toroidal boards [1, on triangular boards [14, 19] and on higher dimensional rectangular boards 24] also fit into this setting.

Surprisingly, formal concepts and prime implicants are also pre-islands in disguise.

Example 2.5. Let $A_{1}, \ldots, A_{n}$ be nonempty sets, and let $\mathcal{I} \subset A_{1} \times \cdots \times A_{n}$. Let us define

$$
\begin{aligned}
U & =A_{1} \times \cdots \times A_{n}, \\
\mathcal{K} & =\left\{B_{1} \times \cdots \times B_{n}: \emptyset \neq B_{i} \subseteq A_{i}, 1 \leq i \leq n\right\} \\
\mathcal{C} & =\{C \in \mathcal{K}: C \subseteq \mathcal{I}\} \cup\{U\},
\end{aligned}
$$

and let $h: U \longrightarrow\{0,1\}$ be the height function given by

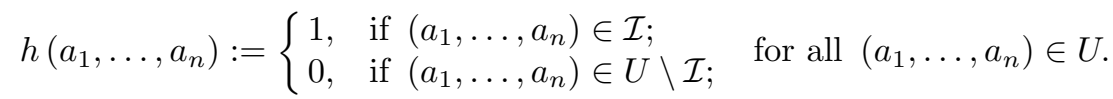

It is easy to see that the pre-islands corresponding to the triple $(\mathcal{C}, \mathcal{K}, h)$ are exactly $U$ and the maximal elements of the poset $(\mathcal{C} \backslash\{U\}, \subseteq)$.

Example 2.6. Let $(G, M, \mathcal{I}), \mathcal{I} \subseteq G \times M$ be a formal context, and let us apply the above construction with $A_{1}=G, A_{2}=M$ and $U=A_{1} \times A_{2}$. Then the proper pre-islands correspond to the concepts of the context $(G, M, \mathcal{I})$ with nonempty extent and intent [10]: the island $B_{1} \times B_{2}$ corresponds to the concept $\left(B_{1}, B_{2}\right)$. 
Example 2.7. Consider the case $A_{1}=\cdots=A_{n}=\{0,1\}$ in Example 2.5. Then the height function $h$ is an $n$-ary Boolean function, and it is not hard to check that the pre-islands corresponding to $(\mathcal{C}, \mathcal{K}, h)$ are $U$ and the prime implicants of $h[2$.

Remark 2.8. For any given island domain $(\mathcal{C}, \mathcal{K})$, maximal families of (pre-)islands are realized by injective height functions. To see this, let us assume that $h$ is a noninjective height function, i.e. there exists a number $z$ in the range of $h$ such that $h^{-1}(z)=\left\{s_{1}, \ldots, s_{m}\right\}$ with $m \geq 2$. The following "refinement" procedure constructs another height function $g$ so that every (pre-)island corresponding to $(\mathcal{C}, \mathcal{K}, h)$ is also a (pre-)island with respect to $(\mathcal{C}, \mathcal{K}, g)$. Let $y$ be the largest value of $h$ below $z$ (or $z-1$ if $z$ is the minimum value of the range of $h$ ), and let $w$ be the smallest value of $h$ above $z$ (or $z+1$ if $z$ is the maximum value of the range of $h$ ). For any $u \in U$, we define $g(u)$ by

$$
g(u)=\left\{\begin{aligned}
y+i \frac{w-y}{m+1}, & \text { if } u=s_{i} ; \\
h(u), & \text { if } h(u) \neq z .
\end{aligned}\right.
$$

By repeatedly applying this procedure we obtain an injective height function without losing any pre-islands. Note that injective height functions correspond to linear orderings of $U$ (cf. the last observation of Remark 2.3.

Example 2.9. Let $U$ be a finite projective plane of order $p$, thus $U$ has $m:=p^{2}+p+1$ points. Let $\mathcal{C}=\mathcal{K}$ consist of the whole plane, the lines, the points and the empty set. Then the greatest possible number of pre-islands is $p^{2}+2=m-p+1$. Indeed, as explained in Remark 2.8, the largest systems of pre-islands emerge with respect to linear orderings of $U$. So let us consider a linear order on $U$, and let $\mathbf{0}$ and $\mathbf{1}$ denote the smallest and largest elements of $U$, respectively. In other words, we have $h(\mathbf{0})<h(x)<h(\mathbf{1})$ for all $x \in U \backslash\{\mathbf{0}, \mathbf{1}\}$. Clearly, a line is a pre-island iff it does not contain $\mathbf{0}$, and there are $m-p-1$ such lines. The only other pre-islands are the point 1 and the entire plane, hence we obtain $m-p-1+2=m-p+1$ pre-islands.

It has been observed in [3, 14, 15, that any two islands on a square or triangular grid with respect to a given height function are either comparable or disjoint. This property is formalized in the following definition, which was introduced in [4].

Definition 2.10. A family $\mathcal{H}$ of subsets of $U$ is CD-independent if any two members of $\mathcal{H}$ are either comparable or disjoint, i.e. for all $A, B \in \mathcal{H}$ at least one of $A \subseteq B$, $B \subseteq A$ or $A \cap B=\emptyset$ holds.

Note that CD-independence is also known as laminarity [21, 25. In general, the properties of CD-independence and being a system of pre-islands are independent from each other, as the following example shows.

Example 2.11. Let $U=\{a, b, c, d, e\}$ and $\mathcal{K}=\mathcal{C}=\{\{a, b\},\{a, c\},\{b, d\},\{c, d\}, U\}$. Let us define a height function $h$ on $U$ by $h(a)=h(b)=h(c)=h(d)=1, h(e)=$ 0 . It is easy to verify that every element of $\mathcal{C}$ is a pre-island with respect to this height function, but $\mathcal{C}$ is not CD-independent. On the other hand, consider the CDindependent family $\mathcal{H}=\{\{a, b\},\{c, d\}, U\}$. We claim that $\mathcal{H}$ is not a system of pre-islands. To see this, assume that $h$ is a height function such that the system of pre-islands corresponding to $(\mathcal{C}, \mathcal{K}, h)$ is $\mathcal{H}$. Let us write out the definition of a pre-island for $S=\{a, b\}$ and $S=\{c, d\}$ with $K=U$ :

$$
\begin{aligned}
& \min (h(a), h(b))>\min h(U) ; \\
& \min (h(c), h(d))>\min h(U) .
\end{aligned}
$$

Taking the minimum of these two inequalities, we obtain

$$
\min (h(a), h(b), h(c), h(d))>\min h(U) .
$$


This immediately implies that $\min (h(a), h(c))>\min h(U)$. Since the only element of $\mathcal{K}$ properly containing $\{a, c\}$ is $U$, we can conclude that $\{a, c\}$ is also a pre-island with respect to $h$, although $\{a, c\} \notin \mathcal{H}$.

As CD-independence is a natural and desirable property of islands that was crucial in previous investigations, we will mainly focus on island domains $(\mathcal{C}, \mathcal{K})$ whose systems of pre-islands are CD-independent. We characterize such island domains in Theorem 4.8, and we refer to them as connective island domains (see Definition 4.1).

The most fundamental questions concerning pre-islands are the following: Given an island domain $(\mathcal{C}, \mathcal{K})$ and a family $\mathcal{H} \subseteq \mathcal{C}$, how can we decide if there is a height function $h$ such that $\mathcal{H}$ is the system of pre-islands corresponding to $(\mathcal{C}, \mathcal{K}, h)$ ? How can we find such a height function (if there is one)? Concerning the first question, we give a dry characterization (i.e., a characterization that does not involve height functions and water levels, as described in the Introduction) of systems of pre-islands corresponding to connective island domains in Theorem 4.9, and in Corollary 5.9 we characterize systems of islands corresponding to so-called proximity domains (see Definition 5.7). These results generalize earlier dry characterizations (see, e.g. [3, 14, 15]), since an island domain $(\mathcal{C}, \mathcal{K})$ corresponding to a graph (cf. Example 2.4) is always a connective island domain and also a proximity domain. Concerning the second question, we give a canonical construction for a height function (Definition 3.4), and we prove in Sections 4 and 5 that this height function works for pre-islands in connective island domains and for islands in proximity domains.

\section{Pre-islands And ADMissible Systems}

In this section we present a condition that is necessary for being a system of preislands, which will play a key role in later sections. Although this necessary condition is not sufficient in general, we will use it to obtain a characterization of maximal systems of pre-islands.

Definition 3.1. Let $\mathcal{H} \subseteq \mathcal{C} \backslash\{\emptyset\}$ be a family of sets such that $U \in \mathcal{H}$. We say that $\mathcal{H}$ is admissible (with respect to $(\mathcal{C}, \mathcal{K})$ ), if for every nonempty antichain $\mathcal{A} \subseteq \mathcal{H}$,

$$
\exists H \in \mathcal{A} \text { such that } \forall K \in \mathcal{K}: H \subset K \Longrightarrow K \nsubseteq \bigcup \mathcal{A} \text {. }
$$

Remark 3.2. Let us note that if $\mathcal{H}$ is admissible, then (1) holds for all nonempty $\mathcal{A} \subseteq \mathcal{H}$ (not just for antichains). Indeed, if $\mathcal{M}$ denotes the set of maximal members of $\mathcal{A}$, then $\mathcal{M}$ is an antichain. Thus the admissibility of $\mathcal{H}$ implies that there is $H \in \mathcal{M} \subseteq \mathcal{A}$ such that for all $K \in \mathcal{K}$ with $H \subset K$ we have $K \nsubseteq \bigcup \mathcal{M}=\bigcup \mathcal{A}$.

Obviously, any subfamily of an admissible family is also admissible, provided that it contains $U$. As we shall see later, in some important special cases a stronger version of admissibility holds, where the existential quantifier is replaced by a universal quantifier in (1): for every nonempty antichain $\mathcal{A} \subseteq \mathcal{H}$,

$$
\forall H \in \mathcal{A} \forall K \in \mathcal{K}: H \subset K \Longrightarrow K \nsubseteq \bigcup \mathcal{A} \text {. }
$$

Proposition 3.3. Every system of pre-islands is admissible.

Proof. Let $h: U \rightarrow \mathbb{R}$ be a height function and let $\mathcal{S}$ be the system of pre-islands corresponding to $(\mathcal{C}, \mathcal{K}, h)$. Clearly, we have $\emptyset \notin \mathcal{S}$ and $U \in \mathcal{S}$. Let us assume for contradiction that there exists an antichain $\mathcal{A}=\left\{S_{i}: i \in I\right\} \subseteq \mathcal{S}$ such that (1) does not hold. Then for every $i \in I$ there exists $K_{i} \in \mathcal{K}$ such that $S_{i} \subset K_{i}$ and $K_{i} \subseteq \bigcup_{i \in I} S_{i}$. Since $S_{i}$ is a pre-island, we have

$$
\min h\left(S_{i}\right)>\min h\left(K_{i}\right) \geq \min h\left(\bigcup_{i \in I} S_{i}\right)
$$




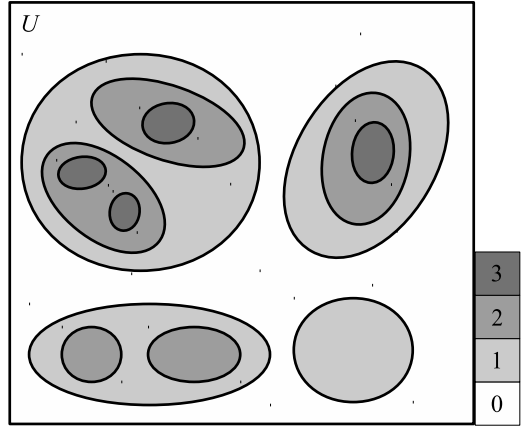

(A) Standard height function

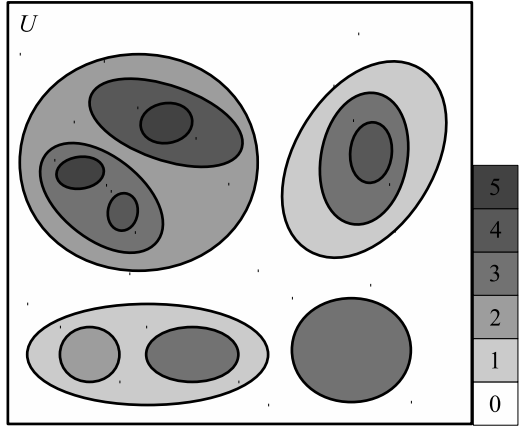

(B) Canonical height function

FIGURE 1. A CD-independent family with two different height functions

for all $i \in I$. Taking the minimum of these inequalities we arrive at the contradiction

$$
\min \left\{\min h\left(S_{i}\right) \mid i \in I\right\}>\min h\left(\bigcup_{i \in I} S_{i}\right) .
$$

The converse of Proposition 3.3 is not true in general: it is straightforward to verify that the family $\mathcal{H}$ considered in Example 2.11 is admissible, but, as we have seen, it is not a system of pre-islands. However, we will prove in Proposition 3.6 that for every admissible family $\mathcal{H}$, there exists a height function such that the corresponding system of pre-islands contains $\mathcal{H}$. First we give the construction of this height function, and we illustrate it with some examples.

Definition 3.4. Let $\mathcal{H} \subseteq \mathcal{C}$ be an admissible family of sets. We define subfamilies $\mathcal{H}^{(i)} \subseteq \mathcal{H}(i=0,1,2, \ldots)$ recursively as follows. Let $\mathcal{H}^{(0)}=\{U\}$. For $i>0$, if $\mathcal{H} \neq \mathcal{H}^{(0)} \cup \cdots \cup \mathcal{H}^{(i-1)}$, then let $\mathcal{H}^{(i)}$ consist of all those sets $H \in \mathcal{H} \backslash\left(\mathcal{H}^{(0)} \cup \cdots \cup \mathcal{H}^{(i-1)}\right)$ that have the following property:

$$
\forall K \in \mathcal{K}: H \subset K \Longrightarrow K \nsubseteq \bigcup\left(\mathcal{H} \backslash\left(\mathcal{H}^{(0)} \cup \cdots \cup \mathcal{H}^{(i-1)}\right)\right) \text {. }
$$

Since $\mathcal{H}$ is finite and admissible, after finitely many steps we obtain a partition $\mathcal{H}=$ $\mathcal{H}^{(0)} \cup \cdots \cup \mathcal{H}^{(r)}$ (cf. Remark 3.2). The canonical height function corresponding to $\mathcal{H}$ is the function $h_{\mathcal{H}}: U \rightarrow \mathbb{N}$ defined by

$$
h_{\mathcal{H}}(x):=\max \left\{i \in\{1, \ldots, r\}: x \in \bigcup \mathcal{H}^{(i)}\right\} \text { for all } x \in U
$$

Observe that every $\mathcal{H}^{(i)}$ consists of some of the maximal members of $\mathcal{H} \backslash\left(\mathcal{H}^{(0)} \cup\right.$ $\left.\cdots \cup \mathcal{H}^{(i-1)}\right)=\mathcal{H}^{(i)} \cup \cdots \cup \mathcal{H}^{(r)}$. However, if $\mathcal{H}$ satisfies 2 for all antichains $\mathcal{A} \subseteq \mathcal{H}$, then the word "some" can be replaced by "all" in the previous sentence, and in this case $h_{\mathcal{H}}$ can be computed just from $\mathcal{H}$ itself, without making reference to $\mathcal{K}$. To illustrate this, let us consider a CD-independent family $\mathcal{H}$. Clearly, for every $u \in U$, the set of members of $\mathcal{H}$ containing $u$ is a finite chain. The standard height function of $\mathcal{H}$ assigns to each element $u$ the length of this chain, i.e. one less than the number of members of $\mathcal{H}$ that contain $u$. (Note that the definition of a standard height function in [17] differs slightly from ours.) It is easy to see that if $\mathcal{H}$ satisfies (2), then the canonical height function of $h$ coincides with the standard height function. However, in general the two functions might be different. Figure 1 represents the standard and the canonical height functions for the same CD-independent family, with greater heights indicated by darker colors. We can see from Figure $1 \mathrm{~b}$ that only two of the four maximal members of $\mathcal{H} \backslash\{U\}$ belong to $\mathcal{H}^{(1)}$, thus 2 fails here. (In order to make the picture comprehensible, only members of $\mathcal{C}$ are shown, although $\mathcal{K}$ is also 
needed to determine $h_{\mathcal{H}}$ (Figure $1 \mathrm{~b}$ ). On the other hand, the standard height function (Figure 1a can be read directly from the figure.)

The next example shows that there exist CD-independent systems of pre-islands for which the standard height function is not the right choice. However, in Section 5 we will see that for a wide class of island domains, including those corresponding to graphs (cf. Example 2.4), the standard height function is always appropriate.

Example 3.5. Let $U=\{a, b, c, d\}, \mathcal{C}=\{A, B, U\}$ and $\mathcal{K}=\{A, B, U, K\}$, where $A=\{a\}, B=\{b, c\}$ and $K=\{a, c\}$. Then the family $\mathcal{H}=\{A, B, U\}$ is admissible; the corresponding partition is $\mathcal{H}^{(0)}=\{U\}, \mathcal{H}^{(1)}=\{B\}, \mathcal{H}^{(2)}=\{A\}$, and the canonical height function is given by $h_{\mathcal{H}}(a)=2, h_{\mathcal{H}}(b)=h_{\mathcal{H}}(c)=1, h_{\mathcal{H}}(d)=0$. It is straightforward to verify that $\mathcal{H}$ is the system of pre-islands corresponding to $\left(\mathcal{C}, \mathcal{K}, h_{\mathcal{H}}\right)$. However, the standard height function assigns the value 1 to $a$, and thus $A$ is not a pre-island with respect to the standard height function of $\mathcal{H}$.

Proposition 3.6. If $\mathcal{H} \subseteq \mathcal{C}$ is an admissible family of sets and $h_{\mathcal{H}}$ is the corresponding canonical height function, then every member of $\mathcal{H}$ is a pre-island with respect to $\left(\mathcal{C}, \mathcal{K}, h_{\mathcal{H}}\right)$.

Proof. Let $\mathcal{H} \subseteq \mathcal{C}$ be admissible, and let us consider the partition $\mathcal{H}=\mathcal{H}^{(0)} \cup \cdots \cup \mathcal{H}^{(r)}$ given in Definition 3.4. For each $H \in \mathcal{H}$, there is a unique $i \in\{1, \ldots, r\}$ such that $H \in \mathcal{H}^{(i)}$, and we have $\min h_{\mathcal{H}}(H) \geq i$ by (4). Using this observation it is straightforward to verify that $H$ is indeed a pre-island with respect to $\left(\mathcal{C}, \mathcal{K}, h_{\mathcal{H}}\right)$.

As an immediate consequence of Propositions 3.3 and 3.6 we have the following corollary.

Corollary 3.7. A subfamily of $\mathcal{C}$ is a maximal system of pre-islands if and only if it is a maximal admissible family.

We have seen in Example 2.11 that it is possible that a subset of a system of preislands is not a system of pre-islands. The notion of admissibility allows us to describe those situations where this cannot happen.

Proposition 3.8. The following two conditions are equivalent for any island domain $(\mathcal{C}, \mathcal{K})$ :

(i) Any subset of a system of pre-islands corresponding to $(\mathcal{C}, \mathcal{K})$ that contains $U$ is also a system of pre-islands.

(ii) The systems of pre-islands corresponding to $(\mathcal{C}, \mathcal{K})$ are exactly the admissible families.

Proof. The implication (ii) $\Longrightarrow$ (i) follows from the simple observation that any subset of an admissible family containing $U$ is also admissible. Assume now that (i) holds. In view of Proposition 3.3 it suffices to prove that every admissible family is a system of pre-islands. Let $\mathcal{H}$ be an admissible family, then Proposition 3.6 yields a system of pre-islands containing $\mathcal{H}$. Using (i) we can conclude that $\mathcal{H}$ is a system of preislands.

\section{CD-INDEPENDENCE AND CONNECTIVE ISLAND DOMAINS}

As we have seen in Example 2.11, a system of pre-islands is not necessarily CDindependent. In this section we present a condition that characterizes those island domains $(\mathcal{C}, \mathcal{K})$ whose systems of pre-islands are $\mathrm{CD}$-independent, and we will prove that admissibility is necessary and sufficient for being a systems of pre-islands in this case. 


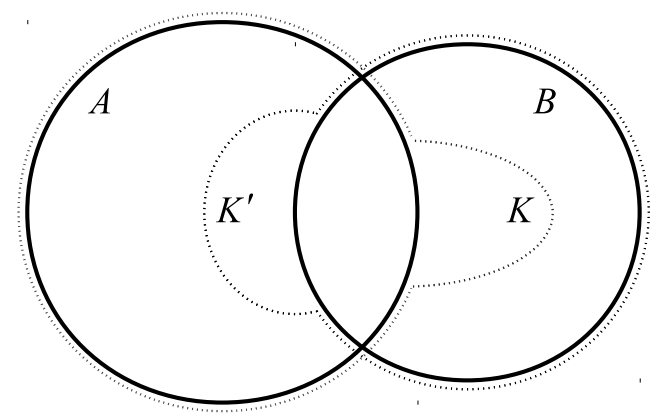

FiguRE 2. Illustration to the definition of an island domain

Definition 4.1. An island domain $(\mathcal{C}, \mathcal{K})$ is a connective island domain if

$$
\forall A, B \in \mathcal{C}:(A \cap B \neq \emptyset \text { and } B \nsubseteq A) \Longrightarrow \exists K \in \mathcal{K}: A \subset K \subseteq A \cup B .
$$

Remark 4.2. Observe that if $A \subset B$, then (5) is satisfied with $K=B$. Thus it suffices to require (5) for sets $A, B$ that are not comparable or disjoint. In this case, by switching the role of $A$ and $B$, we obtain that there is also a set $K^{\prime} \in \mathcal{K}$ such that $B \subset K^{\prime} \subseteq A \cup B$ (see Figure 2).

Remark 4.3. The terminology is motivated by the intuition that the set $K$ in Definition 4.1 somehow connects $A$ and $B$. Let us note that if $(\mathcal{C}, \mathcal{K})$ corresponds to a graph, as in Example 2.4 then $(\mathcal{C}, \mathcal{K})$ is a connective island domain. Furthermore, it is not difficult to prove that if $(\mathcal{C}, \mathcal{K})$ is a connective island domain with $\mathcal{C}=\mathcal{K}$, then (5) is equivalent to the fact that the union of two overlapping members of $\mathcal{K}$ belongs to $\mathcal{K}$ (see $(9)$ in Section 5), which is an important property of connected sets.

We will prove that pre-islands corresponding to connective island domains are not only CD-independent, but they also satisfy the following stronger independence condition, usually called $\mathrm{CDW}$-independence, which was introduced in [6].

Definition 4.4. A family $\mathcal{H} \subseteq \mathcal{P}(U)$ is weakly independent (see [5]) if

$$
H \subseteq \bigcup_{i \in I} H_{i} \Longrightarrow \exists i \in I: H \subseteq H_{i}
$$

holds for all $H \in \mathcal{H}, H_{i} \in \mathcal{H}(i \in I)$. If $\mathcal{H}$ is both CD-independent and weakly independent, then we say that $\mathcal{H}$ is $\mathrm{CDW}$-independent.

Remark 4.5. Let $\mathcal{H} \subseteq \mathcal{P}(U)$ be a CD-independent family, and let $H \in \mathcal{H}$. Let $M_{1}, \ldots, M_{m}$ be those elements of $\mathcal{H}$ that are properly contained in $H$ and are maximal with respect to this property. Then $M_{1}, \ldots, M_{m}$ are pairwise disjoint, and $M_{1} \cup \cdots \cup M_{m} \subseteq H$. Weak independence of $\mathcal{H}$ is equivalent to the fact that this latter containment is strict for every $H \in \mathcal{H}$. In particular, in the definition of weak independence it suffices to require [6] for pairwise disjoint sets $H_{i}$.

Lemma 4.6. If $(\mathcal{C}, \mathcal{K})$ is a connective island domain, then every admissible subfamily of $\mathcal{C}$ is $\mathrm{CDW}$-independent.

Proof. Let $(\mathcal{C}, \mathcal{K})$ be a connective island domain, and let $\mathcal{H} \subseteq \mathcal{C}$ be an admissible family. If $A, B \in \mathcal{H}$ are neither comparable nor disjoint, then (5) and Remark 4.2 show that $\mathcal{A}:=\{A, B\}$ is an antichain for which (1) does not hold (see Figure 2). Thus $\mathcal{H}$ is CD-independent.

To prove that $\mathcal{H}$ is also CDW-independent, we apply Remark 4.5. Let us assume for contradiction that $M_{1} \cup \cdots \cup M_{m}=H$ for pairwise disjoint sets $M_{1}, \ldots, M_{m} \in$ 
$\mathcal{H}(m \geq 2)$ and $H \in \mathcal{H}$. Since $M_{i} \subset H \in \mathcal{K}$ and $H \subseteq M_{1} \cup \cdots \cup M_{m}$ for $i=1, \ldots, m$, we see that (1) fails for the antichain $\mathcal{A}:=\left\{M_{1}, \ldots, M_{m}\right\}$, contradicting the admissibility of $\mathcal{H}$.

As the next example shows, a CDW-independent family in a connective island domain is not necessarily admissible.

Example 4.7. Let us consider the same sets $U, A, B$ and $K$ as in Example 3.5 . and let $\mathcal{C}=\{A, B, U\}$ and $\mathcal{K}=\{A, B, U, K, L\}$, where $L=\{a, b, c\}$. Then $(\mathcal{C}, \mathcal{K})$ is a connective island domain and $\{A, B, U\}$ is $\mathrm{CDW}$-independent, but it is not admissible (hence not a system of pre-islands).

Theorem 4.8. The following three conditions are equivalent for any island domain $(\mathcal{C}, \mathcal{K})$ :

(i) $(\mathcal{C}, \mathcal{K})$ is a connective island domain.

(ii) Every system of pre-islands corresponding to $(\mathcal{C}, \mathcal{K})$ is $\mathrm{CD}$-independent.

(iii) Every system of pre-islands corresponding to $(\mathcal{C}, \mathcal{K})$ is $\mathrm{CDW}$-independent.

Proof. It is obvious that (iii) $\Longrightarrow$ (ii)

To prove that (ii) $\Longrightarrow(\mathrm{i})$, let us assume that $(\mathcal{C}, \mathcal{K})$ is not a connective island domain. Then there exist $A, B \in \mathcal{C}$ that are not comparable or disjoint such that there is no $K \in \mathcal{K}$ with $A \subset K \subseteq A \cup B$. We define a height function $h: U \rightarrow \mathbb{N}$ as follows:

$$
h(x):= \begin{cases}2, & \text { if } x \in B ; \\ 1, & \text { if } x \in A \backslash B \\ 0, & \text { if } x \notin A \cup B\end{cases}
$$

We claim that both $A$ and $B$ are pre-islands with respect to $(\mathcal{C}, \mathcal{K}, h)$. This is clear for $B$, as $\min h(K) \leq 1$ for any proper superset $K$ of $B$. On the other hand, our assumption implies that for any $K \supset A$ we have $K \nsubseteq A \cup B$, hence $\min h(K)=0<$ $\min h(A)=1$, thus $A$ is indeed a pre-island. Since $A$ and $B$ are not CD, the system of pre-islands corresponding to $(\mathcal{C}, \mathcal{K}, h)$ is not $\mathrm{CD}$-independent.

Finally, for the implication (i) $\Longrightarrow($ iii) assume that $(\mathcal{C}, \mathcal{K})$ is a connective island domain and $\mathcal{S}$ is a system of pre-islands corresponding to $(\mathcal{C}, \mathcal{K})$. By Proposition 3.3. $\mathcal{S}$ is admissible, and then Lemma 4.6 shows that $\mathcal{S}$ is CDW-independent.

Our final goal in this section is to prove that if $(\mathcal{C}, \mathcal{K})$ is a connective island domain, then the systems of pre-islands are exactly the admissible subfamilies of $\mathcal{C}$. Recall that this is not true in general if $(\mathcal{C}, \mathcal{K})$ is not a connective island domain (see Example 2.11), but the two notions coincide for maximal families (Corollary 3.7).

Theorem 4.9. If $(\mathcal{C}, \mathcal{K})$ is a connective island domain, then a subfamily of $\mathcal{C}$ is a system of pre-islands if and only if it is admissible.

Proof. We have already seen in Proposition 3.3 that every system of pre-islands is admissible. Let us now assume that $(\mathcal{C}, \mathcal{K})$ is a connective island domain and let $\mathcal{H} \subseteq \mathcal{C}$ be admissible. From Lemma 4.6 it follows that $\mathcal{H}$ is $\mathrm{CDW}$-independent. Let $\mathcal{S}$ be the system of pre-islands corresponding to $\left(\mathcal{C}, \mathcal{K}, h_{\mathcal{H}}\right)$, where $h_{\mathcal{H}}$ is the canonical height function of $\mathcal{H}$ (see Definition 3.4). Then $\mathcal{S}$ is also CDW-independent by Theorem 4.8, From Proposition 3.6 it follows that $\mathcal{H} \subseteq \mathcal{S}$, and we are going to prove that we actually have $\mathcal{H}=\mathcal{S}$.

Suppose for contradiction that there exists $S \in \mathcal{S}$ such that $S \notin \mathcal{H}$. Since $\mathcal{H}$ is CD-independent and finite, the members of $\mathcal{H}$ that contain $S$ form a nonempty finite chain. Denoting the least element of this chain by $H$, we have $S \subset H$, as $S \notin \mathcal{H}$. Let $M_{1}, \ldots, M_{m}$ denote those elements of $\mathcal{H}$ that are properly contained in $H$ and are maximal with respect to this property (if there are such sets). Clearly, $M_{1}, \ldots, M_{m}$ 


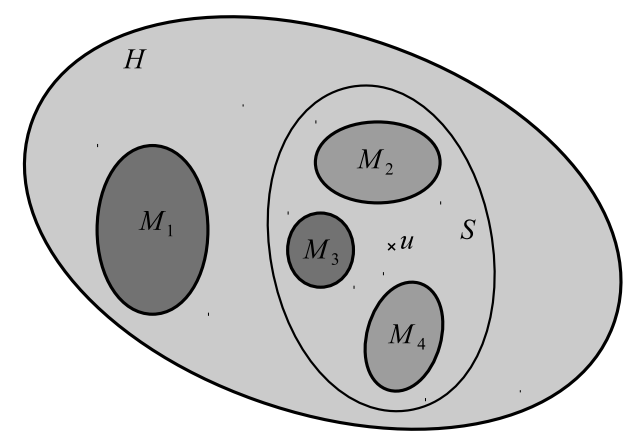

Figure 3. Illustration to the proof of Theorem 4.9

are pairwise disjoint, and $M_{1} \cup \cdots \cup M_{m} \subset H$, since $\mathcal{H}$ is CDW-independent (see Remark 4.5.

We claim that $S \nsubseteq M_{1} \cup \cdots \cup M_{m}$. Assuming on the contrary that $S \subseteq M_{1} \cup \cdots \cup M_{m}$, the CDW-independence of $\mathcal{S}$ implies that there is an $i \in\{1, \ldots, m\}$ such that $S \subseteq M_{i}$. However, this contradicts the minimality of $H$.

Any two elements of $H \backslash\left(M_{1} \cup \cdots \cup M_{m}\right)$ are contained in exactly the same members of $\mathcal{H}$, therefore $h_{\mathcal{H}}$ is constant, say constant $c$, on this set (see Figure 3 , cf. also Figure 1b. On the other hand, if $x \in M_{1} \cup \cdots \cup M_{m}$, then clearly we have $h_{\mathcal{H}}(x) \geq c$, hence $\min h_{\mathcal{H}}(H)=c$. Since $S$ is not covered by the sets $M_{i}$, it contains a point $u$ from $H \backslash\left(M_{1} \cup \cdots \cup M_{m}\right)$, therefore $\min h_{\mathcal{H}}(S)=h(u)=c$. Thus we have $S \subset H \in \mathcal{K}$ and $\min h_{\mathcal{H}}(S)=\min h_{\mathcal{H}}(H)$, contradicting that $S$ is a pre-island with respect to $\left(\mathcal{C}, \mathcal{K}, h_{\mathcal{H}}\right)$.

The maximum number of (pre-)islands certainly depends on the structure of the island domain $(\mathcal{C}, \mathcal{K})$. Härtel [11 proved that the maximum number of rectangular islands on a $1 \times n$ board is $n$, and Czédli [3] generalized this result by showing that the maximum number of rectangular islands on an $n \times m$ board is $\lfloor(m n+m+n-1) / 2\rfloor$. Although these are the only cases where the exact value is known, there are estimates in several other cases [1, 14, 15, 20, 24. In full generality, we have the following upper bound.

Theorem 4.10. If $(\mathcal{C}, \mathcal{K})$ is a connective island domain and $\mathcal{S}$ is a system of preislands corresponding to $(\mathcal{C}, \mathcal{K})$, then $|\mathcal{S}| \leq|U|$.

Proof. Let $(\mathcal{C}, \mathcal{K})$ be a connective island domain and let $\mathcal{S} \subseteq \mathcal{C} \backslash\{\emptyset\}$ be a system of pre-islands corresponding to $(\mathcal{C}, \mathcal{K})$. By Theorem $4.8, \mathcal{S}$ is $\mathrm{CDW}$-independent, and hence $\mathcal{S} \cup\{\emptyset\}$ is also CDW-independent. From the results of [6] it follows that every maximal CDW-independent subset of $\mathcal{P}(U)$ has $|U|+1$ elements. Thus we have $|\mathcal{S}|+1 \leq|U|+1$.

Observe that the above mentioned result of Härtel shows that the bound obtained in Theorem 4.10 is sharp.

\section{ISLANDS AND PROXIMITY DOMAINS}

In this section we investigate islands, and we give a characterization of systems of islands corresponding to island domains $(\mathcal{C}, \mathcal{K})$ satisfying certain natural conditions. We define a binary relation $\delta \subseteq \mathcal{C} \times \mathcal{C}$ that expresses the fact that a set $B \in \mathcal{C}$ is in some sense close to a set $A \in \mathcal{C}$ :

$$
A \delta B \Leftrightarrow \exists K \in \mathcal{K}: \quad A \preceq K \text { and } K \cap B \neq \emptyset .
$$


Remark 5.1. Let us note that the relation $\delta$ is not always symmetric. As an example, consider a directed graph, and let $\mathcal{C}=\mathcal{K}$ consist of $U$ and of those sets $S$ of vertices that have a source. (By a source of a set $S$ we mean a vertex $s \in S$ from which all other vertices of $S$ can be reached by a directed path that lies entirely in $S$.) It is easy to verify that in the graph $a \rightarrow b \rightarrow c \leftarrow d \leftarrow e$ we have $A \delta B$ but not $B \delta A$ for the sets $A=\{a, b\}$ and $B=\{c, d\}$.

Definition 5.2. We say that $A, B \in \mathcal{C}$ are distant if neither $A \delta B$ nor $B \delta A$ holds. Obviously, in this case $A$ and $B$ are also incomparable (in fact, disjoint), whenever $A, B \neq \emptyset$. A nonempty family $\mathcal{H} \subseteq \mathcal{C}$ will be called a distant family, if any two incomparable members of $\mathcal{H}$ are distant.

Remark 5.3. It is not difficult to verify that relation $\delta$ satisfies the following properties for all $A, B, C \in \mathcal{C}$ whenever $B \cup C \in \mathcal{C}$ :

$$
\begin{aligned}
A \delta B & \Rightarrow B \neq \emptyset ; \\
A \cap B \neq \emptyset & \Rightarrow A \delta B ; \\
A \delta(B \cup C) & \Leftrightarrow(A \delta B \text { or } A \delta C) .
\end{aligned}
$$

Lemma 5.4. If $\mathcal{H} \subseteq \mathcal{C}$ is a distant family, then $\mathcal{H}$ is $\mathrm{CDW}$-independent. Moreover, if $U \in \mathcal{H}$, then $\mathcal{H}$ is admissible.

Proof. Let $\mathcal{H} \subseteq \mathcal{C}$ be a distant family, then $\mathcal{H}$ is clearly CD-independent; moreover, it is easy to show using Remark 4.5 that $\mathcal{H}$ is CDW-independent.

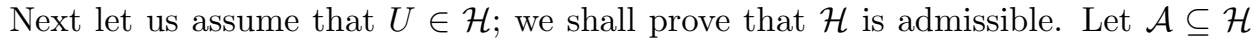
be an antichain and let $H \in \mathcal{A}$. If $K \in \mathcal{K}$ contains $H$ properly, then there is a cover $K_{1} \in \mathcal{K}$ of $H$ such that $H \prec K_{1} \subseteq K$. Since all members of $\mathcal{A} \backslash\{H\}$ are distant from $H$, none of them can intersect $K_{1}$, and therefore we have $K_{1} \nsubseteq \bigcup \mathcal{A}$, and hence $K \nsubseteq \bigcup \mathcal{A}$.

Remark 5.5. Note that we have proved that $\mathcal{H}$ satisfies (2) for every antichain $\mathcal{A} \subseteq \mathcal{H}$. Thus $h_{\mathcal{H}}$ is the standard height function of $\mathcal{H}$.

Theorem 5.6. Let $(\mathcal{C}, \mathcal{K})$ be a connective island domain and let $\mathcal{H} \subseteq \mathcal{C} \backslash\{\emptyset\}$ with $U \in \mathcal{H}$. If $\mathcal{H}$ is a distant family, then $\mathcal{H}$ is a system of islands; moreover, $\mathcal{H}$ is the system of islands corresponding to its standard height function.

Proof. Let $\mathcal{H} \subseteq \mathcal{C} \backslash\{\emptyset\}$ be a distant family such that $U \in \mathcal{H}$. Applying Lemma 5.4 we obtain that $\mathcal{H}$ is admissible, hence $\mathcal{H}$ is the system of pre-islands corresponding to $\left(\mathcal{C}, \mathcal{K}, h_{\mathcal{H}}\right)$ by Theorem 4.9. Moreover, $h_{\mathcal{H}}$ is the standard height function of $\mathcal{H}$ by Remark 5.5

To finish the proof, we will prove that each $H \in \mathcal{H}$ is actually an island with respect to $\left(\mathcal{C}, \mathcal{K}, h_{\mathcal{H}}\right)$. Suppose that $K \in \mathcal{K}$ is a cover of $H$. The distantness of $\mathcal{H}$ implies that the only members of $\mathcal{H}$ that intersect $K \backslash H$ are the ones that properly contain $H$. Since $h_{\mathcal{H}}$ is the standard height function, $h_{\mathcal{H}}(u)<\min h_{\mathcal{H}}(H)$ follows for all $u \in K \backslash H$.

Definition 5.7. The island domain $(\mathcal{C}, \mathcal{K})$ is called a proximity domain, if it is a connective island domain and the relation $\delta$ is symmetric for nonempty sets, that is

$$
\forall A, B \in \mathcal{C} \backslash\{\emptyset\}: A \delta B \Leftrightarrow B \delta A .
$$

If a relation $\delta$ defined on $\mathcal{P}(U)$ satisfies the three properties of Remark 5.3 and $\delta$ is symmetric for nonempty sets, then $(U, \delta)$ is called a proximity space. The notion apparently goes back to Frigyes Riesz [26, however this axiomatization is due to Vadim A. Efremovich (see [7]). 
Proposition 5.8. If $(\mathcal{C}, \mathcal{K})$ is a proximity domain, then any system of islands corresponding to $(\mathcal{C}, \mathcal{K})$ is a distant system.

Proof. Let $(\mathcal{C}, \mathcal{K})$ be a proximity domain, and let $\mathcal{S}$ be the system of islands corresponding to $(\mathcal{C}, \mathcal{K}, h)$ for some height function $h$. Since $(\mathcal{C}, \mathcal{K})$ is a connective island domain, $\mathcal{S}$ is CD-independent according to Theorem 4.8. Therefore, if $A, B \in \mathcal{S}$ are incomparable, then we have $A \cap B=\emptyset$. Assume for contradiction that $A \delta B$, i.e. that there is a set $K \in \mathcal{K}$ such that $A \prec K$ and $B \cap K \neq \emptyset$. Since $A$ and $B$ are disjoint, there exists an element $b \in(B \cap K) \backslash A$. Similarly, as we have $B \delta A$ by (8), there exists an element $a \in\left(A \cap K^{\prime}\right) \backslash B$ for some $K^{\prime} \in \mathcal{K}$ with $B \prec K^{\prime}$. By making use of the fact that both $A$ and $B$ are islands with respect to $(\mathcal{C}, \mathcal{K}, h)$, we obtain the following contradicting inequalities:

$$
\begin{aligned}
h(b) & <\min h(A) \leq h(a) ; \\
h(a) & <\min h(B) \leq h(b) .
\end{aligned}
$$

From Theorem 5.6 and Proposition 5.8 we obtain immediately the following characterization of systems of islands for proximity domains.

Corollary 5.9. If $(\mathcal{C}, \mathcal{K})$ is a proximity domain, and $\mathcal{H} \subseteq \mathcal{C} \backslash\{\emptyset\}$ with $U \in \mathcal{H}$, then $\mathcal{H}$ is a system of islands if and only if $\mathcal{H}$ is a distant family. Moreover, in this case $\mathcal{H}$ is the system of islands corresponding to its standard height function.

Finally, let us consider the following condition on $(\mathcal{C}, \mathcal{K})$, which is stronger than that of being a connective island domain:

$$
\forall K_{1}, K_{2} \in \mathcal{K}: K_{1} \cap K_{2} \neq \emptyset \Longrightarrow K_{1} \cup K_{2} \in \mathcal{K} .
$$

Observe that if we have a graph structure on $U$, and $(\mathcal{C}, \mathcal{K})$ is a corresponding island domain (cf. Example 2.4), then (9) holds.

Theorem 5.10. Suppose that $(\mathcal{C}, \mathcal{K})$ satisfies condition (9), and assume that for all $C \in \mathcal{C}, K \in \mathcal{K}$ with $C \prec K$ we have $|K \backslash C|=1$. Then $(\mathcal{C}, \mathcal{K})$ is a proximity domain, and pre-islands and islands corresponding to $(\mathcal{C}, \mathcal{K})$ coincide. Therefore, if $\mathcal{H} \subseteq \mathcal{C} \backslash\{\emptyset\}$ and $U \in \mathcal{H}$, then $\mathcal{H}$ is a system of (pre-)islands if and only if $\mathcal{H}$ is a distant family. Moreover, in this case $\mathcal{H}$ is the system of (pre-)islands corresponding to its standard height function.

Proof. Let $A, B \in \mathcal{C} \backslash\{\emptyset\}$ be such that $A \delta B$, i.e. $K \cap B \neq \emptyset$ for some $K \in \mathcal{K}$ with $A \preceq K$. If $A \cap B \neq \emptyset$, then clearly $B \delta A$ holds. Suppose now that $A \cap B=\emptyset$. By our assumption, $K=A \cup\{b\}$ for some $b \in B$. From (9) it follows that $K \cup B \in \mathcal{K}$. Since $B \subset A \cup B=K \cup B \in \mathcal{K}$, there exists a cover $K^{\prime} \in \mathcal{K}$ of $B$ such that $B \prec K^{\prime} \subseteq A \cup B$. Clearly, we have $K^{\prime} \cap A \neq \emptyset$, hence $B \delta A$, and this proves that the relation $\delta$ is symmetric. Condition (9) is stronger than $(5)$, therefore $(\mathcal{C}, \mathcal{K})$ is a proximity domain.

From our assumptions it is trivial that every pre-island with respect to $(\mathcal{C}, \mathcal{K})$ is also an island. The last two statements follow then from Corollary 5.9.

Corollary 5.11. Let $G$ be a graph with vertex set $U$; let $(\mathcal{C}, \mathcal{K})$ be an island domain corresponding to $G$ (cf. Example 2.4), and let $\mathcal{H} \subseteq \mathcal{C} \backslash\{\emptyset\}$ with $U \in \mathcal{H}$. Then $\mathcal{H}$ is a system of (pre-)islands if and only if $\mathcal{H}$ is distant; moreover, in this case $\mathcal{H}$ is the system of (pre-)islands corresponding to its standard height function.

\section{CONCLUding REMARKS AND AN ALTERNATIVE FRAMEWORK}

We introduced the notion of a (pre-)island corresponding to an island domain $(\mathcal{C}, \mathcal{K})$, where $U \in \mathcal{C} \subseteq \mathcal{K} \subseteq \mathcal{P}(U)$ for a nonempty finite set $U$. We described island domains $(\mathcal{C}, \mathcal{K})$ having $\mathrm{CD}$-independent systems of pre-islands, and we characterized systems of (pre-)islands for such island domains. In the general case, when 
no assumption is made on $(\mathcal{C}, \mathcal{K})$, we gave a necessary condition for a family of sets to be a system of pre-islands, and it remains an open problem to find an appropriate necessary and sufficient condition. Nevertheless, we obtained a complete characterization of maximal systems of pre-islands in this general case. Determining the size of these maximal systems of pre-islands for specific island domains $(\mathcal{C}, \mathcal{K})$ has been, and continues to be, a topic of active research.

Before concluding the paper, let us propose another possible approach to define islands. Let $U$ be a nonempty finite set and let $\mathcal{C} \subseteq \mathcal{P}(U)$ with $U \in \mathcal{C}$, as before. We describe the "surroundings" of members of $\mathcal{C}$ by means of a relation $\eta \subseteq U \times \mathcal{C}$, where

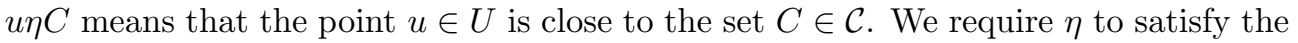
following very natural axiom:

$$
\forall u \in U \forall C \in \mathcal{C}: u \in C \Longrightarrow u \eta C .
$$

Examples of such "point-to-set" proximity relations include closure systems (in particular, topological spaces) with $u \eta C$ if and only if $u$ belongs to the closure of $C$, and graphs with $u \eta C$ if and only if $u$ belongs to the neighborhood of $C$. We shall call a pair $(\mathcal{C}, \eta)$ satisfying $(10)$ an island domain.

For any $C \in \mathcal{C}$, the set $\partial C:=\{u \in U: u \eta C$ and $u \notin C\}$ is the set of points that surround $C$ (note that this is not the usual notion of boundary for topological spaces). Therefore, we define islands corresponding to $(\mathcal{C}, \eta)$ as follows: If $h: U \rightarrow \mathbb{R}$ is a height function and $S \in \mathcal{C}$, then we say that $S$ is an island with respect to $(\mathcal{C}, \eta, h)$, if $h(u)<\min h(S)$ holds for all $u \in \partial S$. This definition is similar in spirit to the definition of an island corresponding to an island domain $(\mathcal{C}, \mathcal{K})$; in fact, it is a generalization of it. To see this, let us consider a pair $(\mathcal{C}, \mathcal{K})$, and let us define $\eta \subseteq U \times \mathcal{C}$ as follows:

$$
u \eta C \Longleftrightarrow \exists K \in \mathcal{K}: C \preceq K \text { and } u \in K .
$$

It is easy to verify that the islands corresponding to $(\mathcal{C}, \eta)$ are exactly the islands corresponding to $(\mathcal{C}, \mathcal{K})$.

Let us now briefly sketch how to adapt the definitions of admissibility, connective island domain and distantness to this setting. We shall say that $\mathcal{H} \subseteq \mathcal{C} \backslash\{\emptyset\}$ is admissible, if $U \in \mathcal{H}$, and for every antichain $\mathcal{A} \subseteq \mathcal{H}$ we have

$$
\exists H \in \mathcal{A} \text { such that } \forall u \in U: u \in \partial H \Longrightarrow u \notin \bigcup \mathcal{A} \text {. }
$$

We call the pair $(\mathcal{C}, \eta)$ a connective island domain if

$$
\forall A, B \in \mathcal{C}:(A \cap B \neq \emptyset \text { and } B \nsubseteq A) \Longrightarrow \exists u \in B \backslash A: u \eta A .
$$

To define distantness, we extend $\eta$ to a "set-to-set" proximity relation $\delta \subseteq \mathcal{C} \times \mathcal{C}$ : for $A, B \in \mathcal{C}$, let $A \delta B$ if and only if there exists a point $u \in B$ with $u \eta A$. Using this relation $\delta$, we can define distant families just as in Definition 5.2 .

Most of the results of this paper remain valid with these new definitions, and the proofs require only minor and quite straightforward modifications. The only exceptions are Lemma 5.4, where we need the extra assumption that $(\mathcal{C}, \eta)$ is a connective island domain, and Theorem 5.10 which cannot be interpreted in this framework, as it refers to $\mathcal{K}$. The following theorem summarizes the main results.

Theorem 6.1. Let $U$ be a nonempty finite set, let $\mathcal{C} \subseteq \mathcal{P}(U)$ with $U \in \mathcal{C}$, and let $\eta \subseteq U \times \mathcal{C}$ satisfy $(10)$.

(i) A family $\mathcal{H} \subseteq \mathcal{C} \backslash\{\emptyset\}$ is contained in a system of islands if and only if $\mathcal{H}$ is admissible.

(ii) A family $\mathcal{H} \subseteq \mathcal{C} \backslash\{\emptyset\}$ is a maximal system of islands if and only if $\mathcal{H}$ is a maximal admissible family. 
(iii) The pair $(\mathcal{C}, \eta)$ is a connective island domain if and only if all systems of islands are $\mathrm{CD}$-independent (equivalently, $\mathrm{CDW}$-independent).

(iv) If $(\mathcal{C}, \eta)$ is a connective island domain, then a family $\mathcal{H} \subseteq \mathcal{C} \backslash\{\emptyset\}$ is a system of islands if and only if $\mathcal{H}$ is admissible.

(v) If $(\mathcal{C}, \eta)$ is a connective island domain and the corresponding relation $\delta$ is symmetric, then a family $\mathcal{H} \subseteq \mathcal{C} \backslash\{\emptyset\}$ is a system of islands if and only if $\mathcal{H}$ is distant and $U \in \mathcal{H}$. Moreover, in this case $\mathcal{H}$ is the system of islands corresponding to its standard height function.

Corollary 6.2. Let $G=(U, E)$ be a connected simple graph, let $\mathcal{C} \subseteq \mathcal{P}(U)$ be a family of connected subsets with $U \in \mathcal{C}$, and let us define $\eta \subseteq U \times \mathcal{C}$ by

$$
u \eta C \Longleftrightarrow u \in C \text { or } \exists v \in C: u v \in E .
$$

Then the following three conditions are equivalent for any $\mathcal{H} \subseteq \mathcal{C} \backslash\{\emptyset\}$ with $U \in \mathcal{H}$ :

(i) $\mathcal{H}$ is a system of islands corresponding to $(\mathcal{C}, \eta)$.

(ii) $\mathcal{H}$ is an admissibly family.

(iii) $\mathcal{H}$ is a distant family.

If these conditions hold, then $\mathcal{H}$ is the system of islands corresponding to its standard height function.

Proof. The fact that $\mathcal{C}$ contains only connected sets ensures that $(\mathcal{C}, \eta)$ is a connective island domain, and it is trivial that $\delta$ is symmetric, hence we can apply Theorem 6.1.

Let us note that in Corollary 6.2 distantness of two sets $A, B \in \mathcal{C}$ means that there is no edge with one endpoint in $A$ and the other endpoint in $B$. Applying this corollary to a square grid (on a rectangular, cylindrical or toroidal board) or to a triangular grid, and letting $\mathcal{C}$ consist of all rectangles, squares or triangles, we obtain the earlier dry characterizations of islands as special cases.

Acknowledgments. Sándor Radeleczki acknowledges that this research was carried out as part of the TAMOP-4.2.1.B-10/2/KONV-2010-0001 project supported by the European Union, co-financed by the European Social Fund.

Eszter K. Horváth and Tamás Waldhauser acknowledge the support of the Hungarian National Foundation for Scientific Research under grant no. K83219. Supported by the European Union and co-funded by the European Social Fund under the project "Telemedicine-focused research activities on the field of Matematics, Informatics and Medical sciences" of project number "TÁMOP-4.2.2.A-11/1/KONV-2012-0073"

Stephan Foldes acknowledges that this work has been co-funded by Marie Curie Actions and supported by the National Development Agency (NDA) of Hungary and the Hungarian Scientific Research Fund (OTKA, contract number 84593), within a project hosted by the University of Miskolc, Department of Analysis. The work was also completed as part of the TAMOP-4.2.1.B.- 10/2/KONV-2010-0001 project at the University of Miskolc, with support from the European Union, co-financed by the European Social Fund.

\section{REFERENCES}

[1] J. Barát, P. Hajnal and E. K. Horváth, Elementary proof techniques for the maximum number of islands, European J. Combin. 32 (2011), 276-281.

[2] Y. Crama and P.L. Hammer, Boolean functions. Theory, algorithms, and applications. Encyclopedia of Mathematics and its Applications 142, Cambridge University Press 2011.

[3] G. Czédli, The number of rectangular islands by means of distributive lattices, European J. Combin. 30 (2009), 208-215. 
[4] G. Czédli, M. Hartmann and E. T. Schmidt, CD-independent subsets in distributive lattices, Publ. Math. Debrecen 74 (2009), 127-134.

[5] G. Czédli, A. P. Huhn and E. T. Schmidt, Weakly independent subsets in lattices, Algebra Universalis 20 (1985), 194-196.

[6] G. Czédli and E. T. Schmidt, CDW-independent subsets in distributive lattices, Acta Sci. Math. (Szeged) 75 (2009), 49-53.

[7] V. A. Efremovich, Infinitesimal spaces, Dokl. Akad. Nauk SSSR 76 (1951), 341-343 (In Russian).

[8] S. Foldes and P. L. Hammer, Disjunctive and conjunctive representations in finite lattices and convexity spaces, Discrete Math. 258 (2002), 13-25.

[9] S. Foldes and N. M. Singhi, On instantaneous codes, J. Comb. Inf. Syst. Sci. 31 (2006), 307-316.

[10] B. Ganter and R. Wille, Formal Concept Analysis: Mathematical Foundations, Springer Verlag 1998.

[11] G. Härtel, personal communication (2007).

[12] E. K. Horváth and S. Radeleczki, Notes on CD-independent subsets, Acta Sci. Math. (Szeged) 78 (2012), 3-24.

[13] E. K. Horváth, A. Máder and A. Tepavčević: One-dimensional Czédli-type islands, College Math. J. 42 (2011), 374-378.

[14] E. K. Horváth, Z. Németh and G. Pluhár, The number of triangular islands on a triangular grid, Period. Math. Hungar. 58 (2009), 25-34.

[15] E. K. Horváth, G. Horváth, Z. Németh and Cs. Szabó, The number of square islands on a rectangular sea, Acta Sci. Math. (Szeged) 76 (2010), 35-48.

[16] E. K. Horváth, B. Šešelja and A. Tepavčević, Cut approach to islands in rectangular fuzzy relations, Fuzzy Sets and Systems 161 (2010), 3114-3126.

[17] E. K. Horváth, B. Šešelja and A. Tepavčević, Cardinality of height function's range in case of maximally many rectangular islands - computed by cuts, Cent. Eur. J. Math. 11 (2013), 296-307.

[18] Zs. Lengvárszky, The minimum cardinality of maximal systems of rectangular islands, European J. Combin. 30 (2009), 216-219.

[19] Zs. Lengvárszky, Notes on triangular islands, Acta Sci. Math. 75 (2009), 369-376.

[20] Zs. Lengvárszky, The size of maximal systems of square islands, European J. Combin. 30 (2009), 889-892.

[21] Zs. Lengvárszky and P. P. Pach, A note on rectangular islands: the continuous case, Acta Sci. Math. (Szeged) 77 (2011), 27-34.

[22] A. Máder and G. Makay, The maximum number of rectangular islands, The Teaching of Mathematics 1461 (2011), 31-44.

[23] A. Máder and R. Vajda, Elementary Approaches to the Teaching of the Combinatorial Problem of Rectangular Islands, Int. J. Comput. Math. Learn. 15 (2010), 267-281.

[24] G. Pluhár, The number of brick islands by means of distributive lattices, Acta Sci. Math. (Szeged) 75 (2009), 3-11.

[25] P. P. Pach, G. Pluhár, A. Pongrácz and Cs. Szabó, The possible number of islands on the sea, J. Math. Anal. Appl. 375 (2011), 8-13.

[26] F. Riesz, Stetigkeitsbegriff und abstrakte Mengenlehre, Atti del IV Congresso Internazionale dei Matematici II (1908), 18-24.

(S. Foldes) Tampere University of Technology, PL 553, 33101 Tampere, Finland

E-mail address: stephan.foldes@tut.fi

(E. K. Horváth) Bolyai Institute, University of Szeged, Aradi vértanúk tere 1, H-6720 SzEged, Hungary

E-mail address: horeszt@math.u-szeged.hu

(S. Radeleczki) Institute of Mathematics, University of Miskold, 3515 Miskolc-Egyetemváros, HUNGARY

E-mail address: matradi@uni-miskolc.hu

(T. Waldhauser) Bolyai Institute, University of Szeged, Aradi vértanúk tere 1, H-6720 Szeged, Hungary

E-mail address: twaldha@math.u-szeged.hu 